\title{
LEONOR FINI, MAGA DEL TRASFORMISMO
}

\section{LEONOR FINI, SORCERESS OF TRANSFORMISM}

\author{
Alessandra Scappini. Università degli Studi di Firenze - Italia \\ a.scappini@virgilio.it
}

Riassunto Leonor Fini (Trieste, 1987 - Parigi, 1996) è una pittrice e scrittrice singolare che lavora nel tempo delle avanguardie storiche avvicinandosi all'entourage del surrealismo a Parigi. Il senso del meraviglioso e l'onnipotenza del desiderio che emergono dalle sue storie e dalle sue opere pittoriche sono aspetti comuni all'avanguardia, anche se non ha mai aderito al movimento. Le figure femminili, gli animali totemici e gli ibridi dipinti o descritti sono simbolici e riflettono il suo gusto per il metamorfismo proprio di una personalità e di un alter ego prossimi all'universo fantastico.

Parole chiave Leonor Fini, surrealismo, desiderio, meraviglioso, fantastico.

\begin{abstract}
Leonor Fini (Trieste, 1987 - Paris, 1996) is a singular woman painter and writer who works in the period of historical avant-gardes approaching the entourage of surrealism in Paris. The sense of the marvelous and the omnipotence of desire that emerge from her stories and her pictorial works are common aspects in the vanguard, even if she has never joined the movement. The female figures, totemic animals and hybrids painted or described are symbolic and reflect her taste for the metamorphism of a personality and an alter ego close to the fantastic universe.
\end{abstract}

Keywords Leonor Fini, surrealism, desire, marvelous, fantastic. 


\section{Introducción. Una esistenza vissuta come un'avventura}

Per Leonor Fini (Buenos Aires 1907 - Parigi 1996) la vita diventa un'avventura fin dalla sua infanzia con sorprese che talvolta creano tensioni in ambito parentale, in quanto che il padre Erminio, proveniente da Benevento e proprietario di haciendas in Argentina, dopo l'unione a Trieste con Malvina Braun, che riunisce in sé le origini dalmate della propria figura materna di discendenza tedesca e di quella paterna originaria di Serajevo, assumerà un atteggiamento indisponente e tirannico, tanto che, in disputa legale con la madre per l'affidamento, tenta per due volte di rapirla, tra il 1909 ed il 1910. Sarà costretta proprio per questo a travestirsi da ragazzo in modo da rendersi irriconoscibile o non identificabile, e da questo momento l'ambiguità del camuffamento diventa caratteristica propria del suo percorso di esistenza, come un divertissement, sperimentato per il gusto personale di esibirsi in pubblico, per gioco, quindi, e per necessità, diventando una autentica maestra di metamorfosi, misteriosa e inclassificabile, come sottolinea Ernestina Pellegrini (2009, p. 16) nel suo saggio dedicato all'artista ${ }^{1}$.

La sua formazione si svolge e alimenta nel clima della città triestina, crocevia di culture, avendo vicino la madre e lo zio, Ernesto Braun, un avvocato liberale progressista, intellettuale colto con una spiccata passione bibliofila, e avvicinandosi presto alla pittura, tanto che vi si dedica completamente dopo una carriera scolastica costellata di espulsioni ${ }^{2}$. Influenzata dall'ambiente fervido di artisti come Arturo Nathan, Edmondo Passauro e Bobi Bazlen³, e dopo gli esordi accademici, da autodidatta, a Milano, dove era stata ospitata dalla famiglia Alberti che le commissiona un ritratto, viene introdotta nel clima di Novecento, conoscendo nell'ambito della seconda esposizione del gruppo Achille Funi, di cui diventa allieva e compagna. Collabora con lui, impegnato nell"epica dell'affresco', nell'ambito della IV Triennale Internazionale di arti decorative presso la Villa Reale di Monza e con lui si trasferisce a Roma per alcuni anni, proseguendo la sua ricerca artistica a Parigi, dove viene ospitata da Vittorio Emanuele Barbaroux, nella cui galleria milanese aveva esposto nel 1929. Al di là delle rassegne nel capoluogo lombardo, presenta nei primi

\footnotetext{
${ }^{1 " S i}$ è alimentata nel tempo, dagli anni trenta in poi, la leggenda di un'artista maestra di metamorfosi, eccessiva, misteriosa, inclassificabile. [...]. Una donna maestra di trasformismo e di mascheramenti, una outsider regale, animata da un sogno di restaurazione matriarcale, una donna che volle fare della propria opera e della stessa vita un gioco a nascondino con se stessa, o anche, se preferite, una specie di mosca cieca biografica con gli spettatori/ lettori", (Pellegrini, 2009. In Masau Dan, 2009, p. 16).

2 "Insofferente ai controlli delle scuole e ai rigori edulcorati delle accademie - il suo - porsi al di fuori degli schemi diventa un modello di comportamento e una necessità creativa. Del porto adriatico rifiuta la patina esteriore e borghese, ordinata, pratica, metodica, piuttosto ne ricerca i lati segreti, sotterranei, misteriosi e inquietanti", (Crusvar, 2009. In Masau Dan, 2009, p. 40).

${ }^{3}$ Leonor incontra l'artista metafisico Arturo Nathan nei primi anni venti, mentre frequenta sua sorella Daisy, amica e compagna di scuola, mentre Edmondo Passauro, designer e ritrattista, che in seguito si trasferisce in Belgio, le offre il gusto per la delicatezza del tratto, come grafico e acquarellista, e Bobi Bazlen la alimenta con i suoi racconti.
} 
anni Trenta le sue opere anche in ambito internazionale. È invitata infatti all'Exposition surréaliste des objets presso la Galerie Charles Ratton a Parigi, alla Mostra internazionale surrealista alla New Burlington Galleries a Londra, mentre tiene una personale alla Galleria Julien Levy a New York ${ }^{4}$, dove, nel corso del suo primo viaggio, incontra Pavel Tchelitchew ed Eugène Berman, e partecipa alla esposizione Fantastic Art, Dada and Surrealism curata da Alfred Barr al Moma. Sono gli anni in cui stringe amicizia con Henri Cartier-Bresson, instaura una relazione affettiva con André Pieyre de Mandiargues e conosce Luc Dietrich, Paul Éluard, Max Ernst, Salvador Dalí, Man Ray e Georges Bataille, avvicinandosi al surrealismo. Il sogno di vivere a Parigi per lei si realizza e nell'entourage francese continuerà il suo percorso creativo ${ }^{5}$.

\section{La sfinge enigma e guardiana}

La sua predilezione per il mistero e il suo gusto per la metamorfosi si fondono nell'emblema della sfinge. Nel mimetismo dei frammenti di sottobosco che compaiono in Sphinx Regina dipinto nel 1943 mentre Leonor soggiorna all'Isola del Giglio sembra di intravedere pupille fisse di uno sguardo ibrido, carismatico e misterioso, come una visione attraverso una lente ravvicinata alla terra. Si celano ed al tempo stesso si svelano come se vegliassero senza manifestarsi nel metamorfismo organico dei suoi ambienti onirici che destano sorpresa e meraviglia. $\mathrm{E}^{\prime}$ il medesimo sguardo carismatico e misterioso della sfinge, un topos che popola le sue opere, prediletto come l'androgino, figura simbolica, emblemi del doppio e dell'ambiguità. Si abbandona infatti all'equivoco che crea dubbio e perplessità, al paradosso che suscita spaesamento e stupore, al capriccio che sollecita fascino e ironia concepibile come humour noir. Al di là delle suggestioni surrealiste, dal momento che la sfinge è un ibrido ricorrente, come incrocio e fusione tra essere umano e animale, nelle opere di André Breton ${ }^{6}$, Max Ernst ${ }^{7}$, Salvador Dali ${ }^{8}$, la sua natura enigmatica corrisponde al carattere di Leonor. Rinvia alla sua infanzia,

\footnotetext{
${ }^{4}$ Julien Levy valorizza tra gli anni Trenta e i Quaranta il lavoro creativo di Leonor e anche di altre artiste, come Kay Sage e Frida Kahlo, nella sua galleria a New York. Leonor Fini vi espone nel 1936, medesimo anno di Fantastic Art, Dada and Surrealism inaugurata al Moma.

${ }^{5} \mathrm{Nel}$ corso degli anni Trenta e Quaranta Leonor Fini vive a Parigi con Stanislao Lepri, un diplomatico che lascia la carriera per dedicarsi alla pittura, mentre è vicina ad André Pieyre de Mandiargues, instaurando per un po' di tempo un menage à trois. Quest'ultimo, stimolato anche da Leonor, pubblica i suoi primi testi e più tardi, quando lei si trasferisce a Roma, legge i suoi scritti come emerge dal carteggio pubblicato nel volume L'ombre portée. Cfr. Bibliografia.

6 "'Raffigurare la sfinge come un leone con la testa di una donna era una volta un fatto poetico, penso che una vera mitologia poetica sia in formazione' [...]. La citazione è tratta da un testo scritto da André Breton in favore di De Chirico. [...]. Breton stabilisce un doppio livello di sfingi, cioè quelle all'interno dell'opera (sono i manichini, sono le statue collocate da De Chirico in quelle misteriose piazze) e poi c'è una sfinge fuori dall'opera e forse è la stessa a cui De Chirico pone gli indovinelli. Quindi il mostro è duplicato [...] nella sfinge 'dell'enunciato' e 'dell'enunciazione', pur mantenendo alcune corrispondenze", (Bolelli, Zaccanti (eds.), 2005 - 2006, p. 60.
} 
La sfinge - commenta Federica Moscolin - sembra essere il nume tutelare dell'esistenza della pittrice, segnandola durevolmente sin dall'infanzia, quando cavalcava nel parco del castello di Miramare la sfinge portata dall'Egitto dall'arciduca Massimiliano. Successivamente la sfinge, come un leitmotiv, continua ad accompagnarla non solo nei suoi dipinti, ma anche sotto forma di soprammobili a protezione delle sue abitazioni o sotto forma di statue nei giardini delle sue case di vacanza (Moscolin, 2009. In Masau Dan, 2009, p. 241).

Come afferma la stessa Leonor nella biografia scritta da Peter Webb (2009, p. 102): "Ricordo che volevo essere come una sfinge". Pura fascinazione per lei, al di là di ogni 'scoria mitica', come sottolinea l'amico e compagno per un periodo della sua vita André Pieyre de Mandiargues:

Nella sfinge, ciò che interessa e appassiona Leonor non è affatto il vecchio mito dell'enigma proposto ai passanti, divenuto pagliacciata letteraria, ma solamente il bel mostro, dove l'umano e il felino si fondono in strettissima unione (Mandiargues, cit. in Moscolin, 2009. In Masau Dan, 2009, p. 241).

Nella sua sottile sensualità infatti diventa seduttrice e soffocatrice per l'uomo che la vede simile a un mostro e incarna perfettamente, per Leonor, la sua predilezione per la bellezza, essenza della vita, cosi come i suoi atteggiamenti da esteta commistionati ad un gusto per l'esotismo e per l'ibridazione di culture e di stili. In ogni caso per lei la sfinge è anche guardiana-protettrice della vita, rivelando la sua umanità al di là del senso di minaccia e di morte. Nella veste di 'guardiana della soglia' tra visibile e invisibile, appartiene all'immaginario come l'unicorno o l'ippogrifo della fiaba moderna e si connota nell'opera di Fini per la predilezione del fantastique che per lei diventa l'unica realtà possibile. II fantastico attraverso il quale Leonor Fini si allontana dal verosimile non è più arbitrario. Si tratta di una nuova prospettiva, sfalsata, che non scredita la visione ordinaria. È ciò che l'artista intende quando scrive: 'Il vero fantastico è l'abito del reale' ${ }^{\prime}$.

\footnotetext{
${ }^{7}$ Max Ernst elabora In the Stable of the Sphinx/ Dans l'écurie du sphinx/ Nella stalla della Sfinge di Storia naturale/ Histoire naturelle, (1925 circa), applicando il frottage alla texture, posizionando la carta su vari materiali, listoni di legno, lunghezze di spago, foglie, rete metallica, carta stropicciata, croste di pane e sfregamento della superficie con una matita o un pastello.

${ }^{8}$ Salvador Dalí realizza nel 1939 la Sphinx of Barcelona, conosciuta anche come Shirley Temple, il più giovane mostro sacro del cinema contemporaneo, con la testa dell'attrice - bambina, enfant prodige presa da una fotografia di una rivista, dominata da un pipistrello e con il corpo di una leonessa rossa con seni evidenti e artigli bianchi, circondata da scheletri umani e ossa. Diventa una critica alla sessualizzazione e allo sfruttamento dell'immagine simbolica della bambina stella del cinema, come immagine dell'innocenza.

9 "Per caratterizzare certe opere si preferirà parlare di fantastique: il termine è più vago che non surrealismo, non rinvia ad alcuna scuola precisa ma si applica a tendenze distribuite in epoche diverse. Qualifica un temperamento, una sensibilità, uno stile i cui tratti si ritrovano nei poeti, nei romanzieri, nei drammaturghi che Leonor ha illustrato, nei pittori e nei musicisti che ha amato", (Dedieu, 2009. In Masau Dan, 2009, p. 13).
} 
Nei suoi lavori creativi è circondata da teschi e ossa, assumendo una funzione distruttrice come in Sphinx Amalburga (1942), mostro sadico, ammaliatrice per giovani uomini che sono le sue vittime. Divinité Chtonienne / Deità Ctonia che veglia il sonno di un giovane (1947) esprime l'ambivalenza sessuale e compare dietro un corpo nudo di un androgino, più minacciosa che protettiva, poiché sembra preconizzare la fine nella decadenza, mentre in The Shepherdess of the Sphinxes/ La pastorella delle sfingi (1941), una giovane figura femminile, ambigua per le forme semianimalesche, si pone quale protettrice di sfingi di forme femminee che sembrano immerse in una palude oscura come luogo del mistero. In Small Guardian Sphinx (1943-1944) l'ibrido donna animale è rappresentato in posizione seduta su un sarcofago come un resto archeologico, con la testa che si avvicina all'immagine di un felino, mentre in Small Lonely Sphinx (1948) appare più prossima a una figura umana in atteggiamento meditativo. Sentinella al limite tra maschile e femminile, tra umano e animale, tra mondo e universo sotterraneo, in verità, si configura altresi come emblema del matriarcato, in quanto che nella sua doppia natura esprime l'integrità dell'androgino, il Rebis alchemico e l'energia come potenziale femminile per guidare e organizzare con naturalezza una comunità intenta a svolgere i propri compiti quotidiani.

Da The Shepherdess of the Sphinxes (1941) al suo viaggio in Egitto dieci anni più tardi, Fini ha presentato la sfinge egiziana come una figura matriarcale e umanista. In effetti, la fotografia di Fini scattata da Adrien de Menasce accanto alla Grande Sfinge a Giza rivela il rapporto dell'artista con la creatura trionfante e il suo ruolo, a sua volta, per il surrealismo: Fini posa in modo tale da vedere solo la parte superiore del busto, gli occhi rivolti verso il basso, mentre si mette in scena come l'immagine speculare della sfinge pensosa e protettiva. (Mahon, 2013, p. 5).

Nella melma in cui sono immerse, le sfingi non affondano nel buio, bensi risalgono in superficie per rivelare ciò che hanno visto nel magma, tanto che sembrano tutelate per garantire la conoscenza come rivelazione. La sua visione matriarcale del mito e dell'universo rinvia, allora, alla veggenza tipica dei culti antichi e alle credenze primordiali, alle origini del processo umano, anche se tra le sue creazioni appare talora la componente erotica, traendo suggestioni dal Marchese de Sade, prossima alla sexual jouissance che può spiegare l'interpretazione critica di Gauthier più propensa a promuovere l'immagine della libertà sessuale rispetto all'iconografia materna ${ }^{10}$.

\footnotetext{
10 "Quanto alle sfingi dipinte da Léonor Fini non sono soltanto un simbolo del mistero, ma si può dire che conservino il senso che gli antichi Egizi davano loro, vale a dire quello della forza invincibile del faraone quando attacca i nemici", (Gauthier, 1973, pp. 133, 143-144). Sexual jouissance si riferisce a uno stato di abbandono come nell'estasi mistica per cogliere la visione; è l'inafferrabile estasi sessuale che si ottiene senza l'esperienza fallica, una gioia travolgente paragonabile, secondo Lacan, all'Estasi di Santa Teresa di Gian Lorenzo Bernini. Il godimento è "un termine indefinibile che si riferisce all'orgasmo sessuale" (Kang, 2002, pp. 98, 100), ma, al contrario, è una gioia pura, non fisica, paragonabile a quella spirituale.
} 
Le sue sfingi, così come i suoi scheletri, ossessionano le menti, senza turbarle [...]. II mondo animale e quello umano si mescolano nell'ibridità più assoluta e perfetta. [...] Jean Claude Dedieu fa notare nella sua prefazione all'opera che I'animalità è da sempre segno di bellezza e che, al contrario, la mostruosità è incarnata da deformità specificatamente umane. [...]. La simbologia delle donne - sfingi non fa che intensificare l'ambiguità della sua creazione. Proprio come le donne rasate, le sfingi trasformano un mondo nell'opposto di ciò che vive ed esiste. Si reincarnano per sorvegliare meglio. Hanno migliaia di occhi, e le spalle tonde o i seni scoperti e tesi sono solamente una fragile apparenza per governare un universo che sta per rinascere (Godard, 1998, pp. 28, 51, 62).

Cosi l'acquitrino dipinto in Le bout du monde/ La fine del mondo (1948), diventa alimentatore similmente al liquido amniotico in cui appare una bellezza femminile che tenta di emergere dal fondo,

immerso nell'acqua di uno stagno, o di una palude, fino al busto, circondato da foglie secche galleggianti e teschi di animali con occhi che affliggono lo spettatore in modo inquietante. Ogni effetto è raddoppiato dal riflesso dell'acqua (Masau Dan, Gregorat e Moscolin, 2009, p. 95)

Nel gran mare dipinto, Fini si testimonia di nuovo in presenza, come 'altra' se stessa, ideale o surreale, nella necessità di riemergere, di rinnovarsi esorcizzando la morte, come se la bellezza potesse vincerla. Nello stesso tempo, considerando se stessa come 'altro', sembra ripercorrere le idee del 'doppio' e 'altro' che Simon de Beauvoir espone nel suo libro The Second Sex, pubblicato a Parigi nel 1949, anche se Leonor dice che non si sente femminista, perché evita il confronto con l'vomo e non inalbera il vessillo dell'uguaglianza che invita a paragonare l'essere femminile all'essere maschile. Ognuno per Leonor è diverso, deve essere se stesso e ha la propria individualità senza vere opposizioni, anche se a volte appaiono di nuovo nel contesto sociale, tanto che 'altro' non è concepibile come 'appendice' dell'vomo, ma come 'altro' da se stesso, alter ego nella sua propria diversità.

\section{Lo sguardo felino e panerotico di Leonor}

Il mondo pittorico e narrativo di Leonor è arricchito da esseri bestiali che assumono un significato totemico, poiché concentrano ed esprimono i suoi stati emotivi, le sue ossessioni e le sue ansie. Nella dimensione quotidiana i gatti sostituiscono le sfingi per Leonor, che fin da piccola indica alle amiche di scuola che le sue pupille sono come quelle di un gatto, di cui subisce la fascinazione in quanto nella sua doppia natura di animale sacro nell'antico Egitto tra Bastet, gatto benevolente, e Sekhmet, leonessa malefica, riunisce molteplici significanze tra madre/ deità mostro, terra/ inferno, vita/ morte. Se da un lato può produrre anche effetti castranti ${ }^{11}$, come nell'immagine del

\footnotetext{
11 Il complesso di castrazione, come meccanismo psicologico che si manifesta nell'essere maschile come paura atavica di una punizione e stato di angoscia è associato da Freud alla figura di Edipo che sente la minaccia di essere castrato per il suo desiderio e le sue fantasie erotiche nei confronti della figura materna. Lacan concepisce l'inconscio come un linguaggio e dunque il fallo come significante, che 'nasce dalla promessa di un potere onnipresente, fallocentrico, irraggiungibile', minacciando
} 
pazzo nella carta dei Tarocchi, in cui morde l'uomo raffigurato alle gambe, dall'altro può alimentare la fertilità, come emerge nei suoi romanzi brevi, tra i quali Murmur, scritto nel $1975^{12}$, in cui Leonor narra gli esiti strabilianti e febbricitanti della potenza sconvolgente del desiderio alimentato ed esaudito. In tal caso il gatto diventa un amante perfetto preferito all'vomo, a cui insegnare tutti i segreti dell'amore e in cui confidare per conservare e aumentare l'energia, linfa della vita. Cosi l'unione sessuale del protagonista Murmur con la madre Lucidor, entrambi felini, coincide con I'iniziazione, come incontro con la Grande Madre per la trasformazione di se stessi e la liberazione del desiderio ritornando alle origini. L'itinerario indirizzato alla scoperta della verità per cogliere il senso del meraviglioso indubbiamente comporta nella narrazione anche la presenza del veggente che appartiene sempre al mondo dei felini e che assume un significato oracolare:

il grande consigliere bianco: il divino Eliodoro seduto su un trono formato da esagoni di zaffiri intervallati da inserti di amazzonite bruna, tormalina e opale scuro. [...] Parla di rado e quando parla lo fa in versi arcaici e per enigmi e sentenze. Si dice che sia eterno, o lo si auspica. Non indossa né vestiti né mantello, ma un grande berillo, chiamato Eliodoro come lui, gli ciondola sul petto, ed è difficile capire come faccia a reggersi, dal momento che non si vedono né una catenina né un laccio. Questo gioiello emette suoni simili a quelli di un'arpa d'oro (Fini, [1976], 2014, pp. 32, 39-40).

Leonor preferisce vivere con i gatti, creature superiori per la potenza dello sguardo di cui sembra appropriarsi, tanto che i suoi atteggiamenti, i suoi costumi sono propri di una donna gatta, come si ritrae ne La vita ideale (1950) tra i gatti accovacciati e un ocellotto, che percepisce a distanza ravvicinata il proprio mondo nel suo metamorfico trasformarsi. Veste e cambia mille maschere per indossare quella da felino che le è forse più congeniale, non tanto perché si approssima all'immagine della predatrice quanto per il senso di mistero che promana dalla fascinazione dello sguardo.

I gatti invitano il vento a giocare con loro. Maho, Mine, Moune, Tigrina si stiracchiano in attesa delle desiderate carezze. Danno la caccia al pavone dalle piume di luce, graffiano il viso malinconico della sfinge, poi si fissano a osservare i movimenti veloci di una nuvola nel cielo scolorito; mentre il mistero del loro sguardo verde e allungato evoca sensazioni di viaggio [...]. Vibrissa diventa bruco lanuginoso e Sarah si veste come un ippocampo per trasformarsi in cavallo araldico e chiudersi come un punto. [...]. Gatti-pesci, gatti-otarie, gatti-lucertole, Rinfignina apre le ali da granduchessa, Maoua

entrambi i sessi con il 'complesso di castrazione'. La legge del padre, come effetto del sistema linguistico, in quanto atto di interdizione al godimento pulsionale illimitato del figlio verso la madre, introduce una mancanza, ma è quest'ultima che determina nel figlio altresi il generarsi del desiderio illimitato e la scoperta della propria identità. Attraverso la nominazione linguistica del "padre" si risolve il complesso edipico. In alcune opere, come The Capital Peine/ La pena capitale (1976), Leonor Fini sembra trarre suggerimenti dallle idee lacaniane di natura linguistica.

${ }^{12} \mathrm{Cfr}$. Bibliografia. 
rivela la sua natura inafferrabile mentre Moufti diventa violento, bastardo, brutale (Fini, cit. In Godard, 1998, pp. 73, 81).

Capace di svelare e celare, di vedere nel buio, di ostentare una sensualità sottile senza morbosità come altra faccia della donna guardiana, appare nei lavori come Stryge Amauri (1947), Sphinx Amalburga (1942) e Divinity Chtonienne (1946), protettrice nei confronti dell'essere maschile a volte addormentato o caduto a terra preso da un sonno-sogno.

I gatti - pensa il protagonista di Onéiropompe, un altro romanzo breve da lei pubblicato nel 1978 - come i preromantici, amano le rovine, gli anfratti difficili, i passaggi imprevisti dove corrono le lucertole blu, a cui a volte manca l'estremità della coda (Premuda, 2014. In Fini, [1976], 2014, p. 101).

Crea inoltre costumi da gatto per il teatro ${ }^{13}$, prediletto da Leonor che ama il travestimento tanto da essere stata definita 'maestra', o addirittura, potremmo aggiungere, 'maga', ricordando i suoi abiti sgargianti o i mantelli di piume da donna uccello, ricchi di paillettes e lustrini da lei ideati e indossati durante i mondani balli in maschera nella capitale francese, e le scenografie dagli effetti destabilizzanti come gli ambienti dei suoi romanzi brevi, autentiche 'camere delle meraviglie', che provocano suggestioni e stimolano corrispondenze analogiche.

Se la pittura rappresenta un approccio biologico per Leonor Fini, il tema del gatto rimane per lei una necessità interiore della sua vita. La pittrice lo spiega cosi: 'La realtà, il quotidiano possono rivelarsi strani e anche meravigliosi. Basta aprire gli occhi e posare sulle cose uno sguardo attento per far si che non ci siano più fenomeni abituali o comprensibili' (Godard, 1998, pp. 79 - 83).

Spaesamento, meraviglia, incontro sonno veglia, metamorfismo sono gli ingredienti della produzione pittorica e letteraria di Leonor Fini che, cosi che "porta il lettore a identificarsi con l'io narrante e a perdersi nei meandri della vicenda con lo stesso stupore che prova il protagonista" (Premuda, 2014. In Fini, [1976], 2014, pp. $103,107)^{14}$. Come in Les vases communicants di André Breton, I'onirico diventa l'elemento più rilevante e quindi ogni riferimento spazio temporale della realtà si vanifica per dare luogo alla sospensione e alla vacuità annunciatrice della vanitas. Spesso il paesaggio in cui compaiono le sue sfingi o guardiane, nella "interferenza di generi e specie: umani e non umani e animali, minerali - che - si mescolano nell'anarchia di

\footnotetext{
13 "Dal 1945 ha partecipato come decoratrice in molti balletti, ha lavorato per Balanchine e per Roland Petit. Nel teatro ha realizzato costumi e scenografie per Berenice presso la compagnia J.- L. Barrault, come per Le Mal court di Audiberti, Les Amants puérils di Crommelynck o La Mégère apprivoisée di Shakespeare. Nel 1953 crea i costumi del film di Renato Castellani Roméo e Juliette. Ha anche progettato Tannhäuser per l'Opera di Parigi nel 1963. Le sue creazioni sono sontuose, spesso barocche, e le piace introdurre maschere nelle sue invenzioni per la danza. Ad esempio, nel balletto di Jean Anouilh e Roland Petit, Les demoiselles de la nuit, impose al primo ballerino una maschera felina rossa. Gli autori e i registi la invitano prontamente perché le sue creazioni sono spesso abbaglianti", (Keyser, 2004, p. 33).

14 "Anche mentre dipinge o descrive i gatti con la punta della sua penna attenta e umoristica, Leonor rimane sempre fedele al suo senso del prestigioso, del fantastico e dell'ambiguo. Ama disorientare, inventando altre glorie", (Godard,1998, pp. 79 - 83).
} 
mutazioni imprevedibili e parziali", (Escoubas, 1978, pp. 385 - 387) è magmatico, un luogo acquitrinoso in cui pulsano microrganismi che affondano nell'acqua per riemergere, rinviando al mistero, all'enigma spaesante. II liquido vitale che accoglie ogni essere, amniotico e placentare in relazione al ciclo della vita come generazione, morte e rinascita, appare fluido come le figure di Leonor, "itineranti, vaganti, stra - vaganti", suscettibili di trasformazioni continue, mobili, quindi, proprio perché sempre pronte a modificare il loro corpo delocalizzato ${ }^{15}$ nello spazio non identificabile, che rinvia al carattere dinamico improntato al cambiamento proprio dell'artista, la non fissità della propria identità mutante, per cui ogni barriera o confine si vanificano, e tutto è possibile come nella vita di Leonor disposta alla sorpresa. Pensieri che diventano sogni trascritti cui si dedica per consuetudine come alla corrispondenza epistolare e che svelano un innesto di personaggi reali disposti a vivere il mutamento in spazi labirintici e situazioni spaesanti che rispecchiano la sua personalità, il suo gusto: "Ho bisogno di consumare molte persone e di fare la ruota in pubblico - dice - È un sentimento spettacolare di me stessa che mi rende inquieta e mutevole" (Fini, cit. In Mandiargues and Fini, 2010, p. 148).

Approdo al fantastico o all'onirismo psicanalitico?

Nella pittura di Leonor, in verità, tutto ciò che è visionario assume dimensione quotidiana, emerge alla coscienza e si fa pittura ed al tempo stesso ciò che è reale si bagna di un velo trasparente di onirismo, come se le figure umane e gli oggetti scorressero lungo i meandri del sogno che di nuovo diventa ed è realtà. 'Realismo irreale' sottolinea Jean Cocteau nel 1951 a proposito della sua pittura, 'realismo surreale' aggiunge Jocelyne Godard a distanza di cinquanta anni nel 2001. Combinazione tra lavoro di fantasia e associazione psichica per cui la 'naturalezza' dell'emersione onirica prende forma in pittura utilizzando gli elementi del fantastique che assumono indubbiamente connotazioni visionarie, mentre ogni contraddizione o opposizione talora permane in dialettica alternanza per provocare spaesamento e suscitare una riflessione critica che, in ogni caso, assume i toni dell'ironia più giocosa o del grottesco. Opposizione o fusione, dualità e unità, sono termini che si alternano nell'opera della Fini, come se fosse combattuta da un lato tra il desiderio di raggiungere la totalità per godere della saggezza, ritrovare l'essenza a petto della vanitas dell'esistenza e, dall'altro, il riemergere della scissione, del dualismo, del confronto come opposizione, con l'altro se stesso. L'essere maschile appare nelle opere sempre 'metamorfosato', 'trasformato' per approssimarsi al femminile, talvolta

15 "Così I'unità della figura dell"uno' - commenta Éliane Escoubas, che propone un' interpretazione piuttosto singolare dell'itinerario dell'artista - si dissolve in molteplicità ripetitiva del quasi identico; $c^{\prime}$ è disorganizzazione della figura / corpo, per disorganizzazione della localizzazione, per spostamento decentramento della figura. C'è disorganizzazione del tempo finalizzato, tempo della capitalizzazione, tempo dello sviluppo. Nessuna apparizione di uno sviluppo, di un prima - dopo, no, ma il vagare, la ripetizione, la simultaneità. II disturbo, i 'nontempi' dei processi primari, la non fissazione. Vagare pure: treni e passeggeri (sguardo perso), altalene, giochi di 'vertigini', giochi spaziali", (Escoubas, 1978, pp. 385-387). 
con i tacchi o con gli zoccoli come un fauno, debole di fronte al volto del 'potere femminile', interpretabile come un potenziale creativo e organizzativo, un matriarcato che non è inteso come dominio, ma come atteggiamento naturale per la cura, parentale, tipico di una madre, come protettrice e organizzatrice delle comunità delle origini $^{16}$.

In alcune opere trapela la potenza panerotica del suo sguardo 'felino', appena profuso rispetto all'ossessione, all'allucinazione che può irrompere nel vissuto da un momento all'altro; diventa voyeuristico come espressione della sua divergenza, ammiccante. Éliane Escoubas, che esprime le sue riflessioni in merito al Livre ${ }^{17}$ di Leonor Fini, sottolinea il potenziale energetico dello sguardo, nonchè la fluidità quale caratteristica della mobilità femminile e della reversibilità delle figure-emblemi che compaiono nelle sue opere, distinguendo la sessualità dalla sensualità. E' uno sguardo di compiacimento nei confronti della bellezza intesa come gusto per la diversità che invita alla divergenza, provocazione e persino propensione verso l'eterosessualità e I'omosessualità che il diktat di Breton non ammetteva ${ }^{18}$.

le - sue - capacità di indagare e osservare traspaiono dallo sguardo penetrante e trafittivo, - sottolinea Mario Praz (1979, p. 206) - che può disorientare o ipnotizzare. I celebri occhi di giaietto bruciano, infiammano, divorano: sono gli occhi che un tempo i pallidi inquisitori avrebbero addebitato alle streghe, o che forse gli antichi elleni avrebbero assegnato alle pitonesse, possedute da qualche divinità crudele e gelosa, e ancora i greci e i romani alle sibille, che hanno il dono avvelenato dello sguardo lungo, creature della soglia tra il mondo degli uomini e degli dei, invasate e liminali, dalla vitalità e dalla libertà concesse solo agli esseri straordinari e paventati.

\section{Il doppio}

Ogni figura diventa immagine di se stessa in cui specchiarsi per ricercare ogni volta la propria identità mutevole, il proprio essere come donna e primariamente come persona con narcisismo e autocompiacimento, per cui lo sguardo si contempla nello specchio riflettente, oggetto per lo sdoppiamento, consente la riflessione e la possibilità di conoscere se stessi, di cogliere attraverso l'alter ego i molteplici volti che comportano di perdersi nella relatività o di ritrovarsi nella unità del Tutto, mettendosi alla prova, superando se stessi per raggiungere l'Assoluto.

\footnotetext{
16 "La società immaginaria creata dalla Fini è decisamente matriarcale, nella misura in cui ricrea l'organizzazione spirituale delle società primitive, naturalmente, matriarcali. Tuttavia, questo non è un segno di un' ipotetica superiorità femminile, ma di naturale appartenenza a una cultura antica", (Jelenski, cit. In Godard, 1998, p. 31).

${ }^{17}$ Cfr. Bibliografia.

18 "Breton incarna il paradosso di una dottrina della libertà che avalla un'istituzione autoritaria, intollerante, che fissa un'ortodossia in materia di costumi (ostilità dichiarata all'omosessualità) e di estetica, e che esclude le devianze", (Dedieu, 2009. In Masau Dan, 2009, p. 13).
} 
Il suo desiderio di integrità e fusione è impostato sul 'doppio' come appare in un'opera che Fini elabora nel 1942, in cui propone in primo piano a figura intera la bellezza giovanile di una ninfa, Egeria, che appare dietro, come un'ombra, mascherata e vestita, guardando se stessa. Anche più tardi

Nelle opere - degli anni Cinquanta - ritorna spesso il tema del doppio. Assistiamo, allora, a un'intera serie che riprende questa idea con forza e determinazione. Doppio intangibile, doppio statico, doppio informale, doppi che ci trascinano verso altrettanti interrogativi dai molteplici significati, per imparare a definirsi, a scoprirsi, a opporsi all'Altro interiore, un Altro in cui fondersi per rinascere (Agnati, 2001, p. 8).

La donna di Leonor Fini vive nel suo doppio, come riscoperta - riconoscimento e rinascita - catarsi. Una donna - dea, non dominatrice, ma una guida, guardiana e protettrice del mistero dell'esistenza, anzi sacerdotessa in grado di catturare indizi oracolari.

The Guardian of the Sources/ La guardiana delle sorgenti (1967) guarda I'osservatore che appare in primo piano dietro alcuni recipienti vitrei che potrebbero raccogliere l'acqua come linfa e 'fonte' di vita, o La gardienne à l'oeuf rouge / La guardiana dell'vovo rosso (1955) custodisce nelle sue mani l'uovo cosmico della rubedo alchemica come un oggetto sacro. In altre opere le donne - dee sono raffigurate senza capelli, non tanto perché sono androgini, ma perché

sono l'incarnazione dell'antico ideale della vergine, cioè l'essere femminile - e specialmente nella seconda metà degli anni ' 50 - è chiaramente 'la donna che appartiene a se stessa'. Non è completamente umana, è più della sua controparte di sfinge: la sua pelle è troppo liscia, il suo viso troppo inespressivo, il suo seno senza capezzoli ( Lauter, 1980/1981, p. 46),

avvicinandosi a possibili entità spirituali, simboli di saggezza e conoscenza, attributi di Iside, come emerge in Le penseur / Il pensatore (1954), raffigurato con un mantello simile alle ali di uccello, che rivela le radici della nostra civiltà che purtroppo nel tempo ha limitato i suoi poteri.

Heliodora (1964) presenta, nella sua profusione di colori di ricca consistenza, una nuova dea con la testa rossa, circondata da fiori e frutti; in Hécate (1965) appare con una fragola rossa brillante in contrasto con la rosa bianca sbiadita, che può portare la morte o che raffigura la bella donna senza pietà/ la belle dame sans merci.

The Lady of the Beasts/ La Signora degli Animali, cosi chiamata da Erich Neumann, diventa una divinità simile per l'aspetto all'immagine ctoniana dell'oscurità, della notte, dell'acqua, della terra, come appare nella Divinité chtonienne guettant le sommeil $d^{\prime}$ un jeune homme/Divinità Ctonia che spia il sonno di un giovane (1947), mentre la magmatica palude e la roccia sono i tipici emblemi di fertilità e permanenza.

Leonor assume ogni volta una fisionomia sempre diversa attraverso il travestimento e secondo gli stati d'animo del momento vissuto, quasi a ribadire a se stessa la necessità di vedersi, di scoprirsi nei rivoli più profondi dell'essere per cogliere 
i turbamenti, le inquietudini, le ansie soffuse al di sotto di una mantiglia preziosa o di un eccentrico cappello o della pelle vellutata di una nudità giovanile.

O la nudità o la sovrapposizione cumulativa / ripetitiva di tessuti. - scrive Éliane Escoubas - Sovrapposizione pittorica o fotografica, ripetuta alla 'lettera' nei racconti in cui vengono descritte le molteplicità di oggetti e stoffe il più delle volte in sovrapposizioni. Maschere: diventare un altro, rifiutare la proprietà del volto, ma anche qui in un processo di incompletezza, dispersione, rifiuto della totalizzazione, rifiuto della ripetizione (Escoubas, 1978, pp. $387-388$ ).

Il rinvio degli sguardi è continuo, ma sollecitano la devianza rispetto al modo comune di vedere, e Leonor gioca a travestirsi, per guardarsi, essere guardata, per provocare o divertirsi senza altri scopi con un gusto per il trasformismo come identità mutante, mentre le figure femminili dipinte nelle sue opere rifuggono lo sguardo dell'osservatore, ma non incontrano i suoi occhi e quindi non consentono il riconoscimento.

La figurazione/ripetizione della Fini, lungi dal moltiplicare la rappresentazione della Potenza divina, del potere, del capitale, nell'originalità della concentrazione, fissa lo sguardo nella dispersione utopica [...]. Ecco la dispersione dello sguardo, anche il più apparentemente familiare = anche lo sguardo su figure / corpi non frammentati. Macchina per divertirsi [...]. L' impressione di posa, la suspense di oggetti e le donne dipinte da Leonor Fini ci avvicinano al 'tableau vivant/ quadro vivente' klossowskiano. Oggetto fantastico in cui lo sguardo del riguardante cessa di essere uno sguardo (dello spettatore) (Escoubas, 1978, pp. 381-382).

Il riferimento a Klossowski, interessato a reinventare la figurazione e a ripensare all'idea del simulacro è pertinente, considerando che le sue Wunderkammer narrative esorbitano di oggetti preziosi e testimoniano il suo virtuosismo come gusto per l'eccesso. Leonor possiede, infatti, un proprio universo ricco di elementi contenutistici e formali che sembra 'traboccare' in senso concettuale o ideativo ed esornativo, tanto da approdare quasi ad un virtuosismo stilistico che è stato definito manierista o barocco, e non solo per il suo protendersi verso l'arte del passato, riferendosi al rinascimento maturo ed evocando le espressioni creative tra cinquecento e seicento, ma anche per una singolare attenzione al particolare, preferendo l'azzardo e la bizzarria prossima all'artificio che emerge nell'opera carica di elementi simbolici o emblemi. Attraverso le sue creazioni che gravitano tra reale e immaginario possiamo comprendere che, come spiega Laura Gavioli (2009, p. 265), i surrealisti che ha conosciuto sono stati per lei 'compagni di strada', in base ad un itinerario personale intrapreso in precedenza che trova possibili confluenze sul piano della 'fantasia, intelligenza, velocità di elaborazione, sete di conoscenza e lungimiranza, complessità propria dell'analisi freudiana', ma che si distingue e ne prende le distanze per una propria singolarità di visione.

Da molti classificata come pittrice surrealista perché vicina al circolo di Dalí e Max Ernst, etichetta che comunque lei non ha mai accettato a pieno, 
reputandosi piuttosto un'artista legata al fantastico. [...] uno dei rari esempi di pittore femmina capace d'imporsi per l'originalità delle sue opere. [...] - Per lei - il trasferimento a Parigi è quasi una tappa obbligata. Si avvicina alla cerchia dei surrealisti rivendicando però una sua autonomia dalla corrente, come scrive Cocteau: 'Con lei il soprannaturale diventa naturale'. Nel 1938 comincia a frequentare Max Ernst, Dalí, Man Ray, Georges Bataille e Paul Éluard ma non accetta tutti i principi surrealisti: in lei il realismo incontra il mondo dell'inconscio e la stranezza e produce metamorfosi che sono vicine al Surrealismo. L'anno seguente dipinge La Chambre Noire che vede per soggetti donne vestite di cuoio, singolari, quasi prive di femminilità, figure di un mondo matriarcale e antico che - come abbiamo menzionato - sarà una costante del suo universo pittorico (Premuda, 2003).

In verità, anche se lei nega la sua adesione al movimento di avanguardia,

all'epoca il surrealismo è nell'aria e, senza volerlo, Leonor è surrealista, quasi come è d'avanguardia senza saperlo [...] - è anche vero che - le analogie finiscono dove inizia il 'sistema' surrealista, dove questo diviene un metodo, elabora prescrizioni, dove l'irrazionale è prefabbricato, programmato (Dedieu, 2009. In Masau Dan, 2009, p.13).

Realmente, per quanto riguarda i suoi esponenti, Leonor sottolinea, scrivendo in occasione della esposizione presso il Palazzo dei Diamanti a Ferrara nel 1983, che

le 'affiliazioni' mi hanno sempre infastidito e ho visto la mia differenza più di ogni affinità con la tendenza surrealista ${ }^{19}$. Erano avidi di nuovi giovani per renderli devoti. Anche se i surrealisti hanno aperto e abbattuto delle porte, adolescente, io avevo abbattuto le stesse porte e altre ancora (Fini, 1983, pp. $24-25)$.

\section{Conclusioni}

Il mondo immaginario di Leonor Fini, angelo nero, maga, musa di se stessa, come scrive di lei Jean Claude Dedieu (1983, p. 13), è lucida e precisa espressione della sua interiorità, universo visionario che emerge dal profondo come effluvi che dal gorgo interno giungono in superficie e permangono sulla soglia tra inconscio e conscio in apparizioni mutevoli e metamorfiche. La fantasia le permette di dare forma ad una molteplicità di immagini che riflettono le sue inquietudini, i moti che provengono dagli abissi, ma anche le sue concezioni che si manifestano nell'opera pittorica come teatro della vita 'ulteriore' o 'altra' rispetto al quotidiano ${ }^{20}$. Una pellicola o velo sottile tra sogno e realtà si vanifica e permette il disvelarsi di indizi, cifrari e addirittura di figure

\footnotetext{
19 "Era quasi terrorizzata all'idea che qualcuno potesse darle un'etichetta di neo-romance, surrealista; d'altra parte ha detto no a André Breton che l'avrebbe voluta nel suo gruppo!" (Renzetti, cit. In Masau Dan, 2009, p. 277).

20 "Tra i pennelli e la tela ha posto uno specchio, lo specchio sulla cui superficie a volte passano riflessi gli interni moti della sua fantasia, i capricci, gli aspetti più intimi e affascinanti del suo sentire, e gradatamente li dipinge per noi per tramandarci e farci capire la complessità del suo dramma" (Clerici, 1945. In Fini, 1983, p. 246).
} 
enigmatiche ed oniriche che frequentemente convivono nella realtà quotidiana, come manifestazioni soggette al metamorfismo organico tra reale e surreale, esistenza e sogno, che per i surrealisti è vera vita. Per Leonor campeggiano fantasmatiche su un palco allestito proprio al limite tra conscio e inconscio, tra fantasia e storia personale, esistenza che scorre giorno dopo giorno. Assumono significato simbolico? Sono ombre ricorrenti e prossime alla cancellazione, sono visioni effimere e al di là di rivestire un preciso valore simbolico suscitano stupore come presenze inquietanti e pullulanti nella sfera del pensiero automatico, rinviano alle ossessioni, 'le tenebre del cuore' che dilagano esplorando, come scrive Jean Genet, la nuit du monde (1950. In Fini, 1983, p. 246).

Affascinante e febbrile, fiabesca e misteriosa, spettacolare e teatrale, eccentrica e lunare, ironica e sorprendente, mondana e solitaria, stregonesca e sulfurea, perversa e innocente. Trafittiva e distaccata, individualista e indipendente: per Leonor Fini gli aggettivi si accentuano e si moltiplicano, si collegano formando endiadi e ridondanze, si accumulano per parallelismi e contrapposizioni, (Praz, 1979, p. 206. In Crusvar, 2009, pp. 39 - 40).

scrive Mario Praz, sottolineando le molteplici sfaccettature dell'artista, il suo spirito libero e anticonvenzionale, singolare esempio di mobilità e desiderio di cambiamento, simile anche a Leonora Carrington, Remedios Varo, Kay Sage, Dorothea Tanning, che erano vicine agli artisti surrealisti, ma distanti da ogni classificazione, per la necessità di rinnovare ogni volta se stesse attraverso le proprie energie. Orientarsi verso il surreale come 'paesaggio' del meraviglioso significa per lei aspirare alla libertà di pensiero che va oltre i canoni prestabiliti, alla liberazione da ogni schematismo, pregiudizio e convenzione arbitraria superando e "trasformando la realtà attraverso la fantasia, l'eccesso, la teatralità come necessità vitali", spiega Jean Claude Dedieu (2009. In Masau Dan, 2009, pp. 10-15), per la sua personalità effervescente prossima a una femme fatale/ donna fatale (Strukelj, 2011, pp. 301 - 328) che ha comportato la nascita di una vera mitografia o addirittura di una automitografia.

Maria Masau Dan (2009, p.6) considera che

questa attrazione esercitata dall'apparenza di femme fatale / donna fatale ha certamente giovato alla Fini in termini di celebrità, ma le è nociuto da un punto di vista strettamente artistico, provocando una certa, duratura, diffidenza da parte della critica, soprattutto italiana, che non ha saputo trovare per lei la giusta collocazione nel quadro, pure articolato e vario, del Novecento.

Questo fatto può certamente derivare anche dalla sua preferenza per uno stile di vita selvaggio e un mondo archetipico, per una singolare componente femminile che le permette innanzitutto di riconoscersi nella natura naturans come energia primordiale e di modificare nella vita quotidiana, attraverso una sorprendente 'virtù' metamorfica e camaleontica, qualsiasi sistema arbitrario e convenzionale, suscitando in qualsiasi momento provocazione e meraviglia come personalità indubbiamente 
carismatica e misteriosa che ha preferito percepire sempre la sua esistenza come opera d'arte.

\section{Bibliografía}

Agnati, T. (2001). Leonor Fini. Un realismo soprannaturale/ Leonor Fini. A Supernatural Realism. Art \& Dossier, 16, pp. 6-11.

Bolelli, R.; Zaccanti, A. (eds.), (2005-2006), Dentro le poetiche. II ritorno della Sfinge, ovvero come e qualmente una teoria errata della comunicazione ci seppellirà: intorno e (dentro) a una mostra di Nanni Menetti, in Parol. Quaderni d'arte e di epistemologia, Roma, Italia: Meltemi, 18, p. 60.

Clerici, F. [1945]. Nota critica/Critical Note. In Leonor Fini. (1983), pp. 245 - 246.

Crusvar, L. (2009). Leonor Fini. Simboli, rituali e metamorfosi per una mitologia dell'ambiguità. In Masau Dan, M. (Ed.), (2009), pp. 39 - 54 .

Dedieu, J. C. (1983). Introduzione critica . In Leonor Fini, 1983, pp. 5 - 13.

Dedieu, J. C. (2009). Coté cour, coté jardin. In Masau Dan, M. (Ed.). (2009), pp. 10 - 15.

Escoubas, É. (1978). Fleur de peau (Le Livre de Léonor Fini). Revue d'esthétique, 3/ 4, pp. $381-393$.

Fini, L. (1975). Le livre de Leonor Fini//The Book of Leonor Fini (avec la collaboration de Alvarez, J.). Paris, France: La Guilde du Livre, Éd. Clairefontaine.

Fini, L. (1983). Testimonianza. In Leonor Fini. (1983), p. 25.

Fini, L. [1976]. (2014). Murmur: Fiaba per bambini Pelosi. (Premuda, C. (Ed.)), Salerno, Italia: Arcoiris.

Gauthier, X. (1973). Surrealismo e sessualità. (trans. Traversi, A.). Milano, Italia: Sugar.

Gavioli, L. (2009). A Leonor, i suoi amici. In Masau Dan, M. (Ed.). (2009), p. 265.

Genet, J., (1950). Nota critica. In Leonor Fini. (1983), p. 246.

Godard, J. (1998). Leonor Fini: Le realtà possibili. Milano, Italia: Selene.

Masau Dan, M. (Ed.). (2009). Leonor Fini: L'italienne de Paris. Trieste, Italia: Comune di Trieste.

Mandiargues, A.P. (de) and Fini, L. (2010). Leonor. Fini, L'ombre portée. Correspondance 1932-1945. Paris, France: Le Promeneur-Gallimard.

Jelenski, C. [1967]. Nota critica. In Leonor Fini, 1983, p. 251.

Kang, S. Y. (2002). Tanning's Pictograph: Repossessing Woman's Fantasy. Aurora, 3, pp. 89-104.

Keyser, E. (de). (2004). Leonor Fini. Éloge. Bulletin de la Classe des Beaux Arts. 15, 1/ 6, pp. $29-35$.

Lauter, E. (1980/ 1981). Leonor Fini. Preparing to Meet the Strangers of the New World. Woman's Art Journal, 1, p. 45.

Leonor Fini. (1983). Casalecchio di Reno, Italia: Grafis. 
Mahon, A. (2013). La Feminité triomphante. Surrealism, Leonor Fini and the Sphinx, Dada/ Surrealism, 19, $1, \quad$ p. 2. Recuperato http://ir.uiowa.edu/cgi/wiewcontent.cgi?article $=1274 \&$ context=dadasur

Masau Dan, M. (Ed.). (2009). Leonor Fini. L'italienne de Paris/ Leonor Fini. The Italian of Paris. Trieste, Italia: Comune di Trieste.

Masau Dan, M., Gregorat, S and Moscolin, F. (2009). I dipinti in mostra. Materiali per il catalogo. In Masau Dan, M. (Ed.). (2009), pp. 92 - 97; 98 - 231.

Moscolin, F. (2009). Les livres de Leonor Fini. In Masau Dan, M. (Ed.). (2009), pp. $239-$ 249.

Pellegrini, E. (2009). In maschera ovvero il festival dell'io di Leonor Fini. In Masau Dan, M. (Ed.), pp. $16-24$.

Praz, M. (1979). La casa della vita. Milano, Italia: Adelphi, 1979.

Premuda, C. (2003). Leonor Fini. L'arte oltre il reale. Fucine Mute, 53. Recuperato http://www.fucinemute.it/2003/06/leonor-fini-1\%E2\%80\%ggarte-oltre-il-reale/

Premuda, C. (Ed.) (2014). Leonor Fini scrittrice. In Fini, L. [1976]. (2014), pp. 81-109.

Webb, P. (2009). Sphinx. The Life and Art of Leonor Fini. New York, U. S.: Vendome Press.

Strukelj, V. (2011), Leonor Fini: autoritratti en femme fatale, Bandiera, L. e Saglia, D., De claris mulieribus. Figue e storie femminili nella tradizione europea. Parma, Italia: Monte Università di Parma, pp. 301 - 328.

\section{Cómo referenciar este artículo/How to reference this article:}

Scappini, A. (2018). Leonor Fini, maga del trasformismo. iQUAL. Revista de Género e Igualdad, 1, 201215, doi: 10.6018/iQual.317701

Scappini, A. (2018). Leonor Fini, Maga del trasformismo. [Leonor Fini, sorceress of transformism]. iQUAL. Revista de Género e Igualdad, 1, 201-215, doi: 10.6018/iQual.317701 